\title{
Correction to: FastNBL: fast neighbor lists establishment for molecular dynamics simulation based on bitwise operations
}

\author{
Kun $\mathrm{Li}^{1,2} \cdot$ Shigang $\mathrm{Li}^{1} \oplus \cdot$ Shan Huang ${ }^{1,2} \cdot{\text { Yifeng } \mathrm{Chen}^{3} \cdot \text { Yunquan Zhang }}^{1}$
}

Published online: 26 July 2019

○) Springer Science+Business Media, LLC, part of Springer Nature 2019

\section{Correction to: The Journal of Supercomputing https://doi.org/10.1007/s11227-019-02860-3}

The first main paragraph of the paper contains errors. The correct wording is given below.

Molecular dynamics simulation is widely used to investigate the physical properties, biological structures, or chemical processes for a large number of particles [25]. Many mature molecular dynamics packages, including GROningen MAchine [1,26] (GROMACS), Large-scale Atomic/Molecular Massively Parallel Simulator [10, 23, 29, 30] (LAMMPS), Nanoscale Molecular Dynamics [19, 28] (NAMD) and HOOMD-blue, have been developed exhaustively in recent years. These packages could provide efficient molecular dynamics simulation not available in experiments by rich calculation models and analysis tools in different applications [36]. However, though the molecular dynamics simulation is extensively enabled by these simulation packages, it is still difficult to ignore the computation cost of interaction forces during the simulation which is extremely time-consuming [20]. This is mainly because the force computation requires to calculate the interactions between each pair of particles in the system, giving rise to $\mathrm{O}\left(\mathrm{N}^{2}\right)$ evaluations of the interaction in each time step, where $\mathrm{N}$ is the total number of particles. It is very costly to carry out such a calculation when a great quantity of particles is simulated. Some typical optimization methods are designed for reducing the cost in this process.

The original article can be found online at https://doi.org/10.1007/s11227-019-02860-3.

Shigang Li

shigangli.cs@gmail.com

1 State Key Laboratory of Computer Architecture, Institute of Computing Technology, Chinese Academy of Sciences, Beijing, China

2 School of Computer and Control Engineering, University of Chinese Academy of Sciences, Beijing, China

3 HCST Key Lab at School of EECS, Peking University, Beijing, China 


\section{References}

1. Abraham MJ, Murtola T, Schulz R, Páll S, Smith JC, Hess B, Lindahl E (2015) Gromacs: high performance molecular simulations through multi-level parallelism from laptops to supercomputers. SoftwareX 1:19-25

10. Brown WM, Wang P, Plimpton SJ, Tharrington AN (2011) Implementing molecular dynamics on hybrid high performance computers-short range forces. Comput Phys Commun 182(4):898-911. https://doi.org/10.1016/j.cpc.2010.12.021

19. Jiang W, Hardy DJ, Phillips JC, MacKerell AD Jr, Schulten K, Roux B (2010) High-performance scalable molecular dynamics simulations of a polarizable force field based on classical drude oscillators in namd. J Phys Chem Lett 2(2):87-92

20. Liu W, Schmidt B, Voss G, Müller-Wittig W (2007) Molecular dynamics simulations on commodity GPUs with CUDA. In: International Conference on High-Performance Computing. Springer, pp 185-196

23. Plimpton S (1995) Fast parallel algorithms for short-range molecular dynamics. J Comput Phys 117(1):1-19. https://doi.org/10.1006/jcph.1995.1039

25. Potter D (1973) Computational physics. Wiley

26. Pronk S, Páll S, Schulz R, Larsson P, Bjelkmar P, Apostolov R, Shirts MR, Smith JC, Kasson PM, Van Der Spoel D et al (2013) Gromacs 4.5: a high-throughput and highly parallel open source molecular simulation toolkit. Bioinformatics 29(7):845-854

28. Stone JE, Phillips JC, Freddolino PL, Hardy DJ, Trabuco LG, Schulten K (2007) Accelerating molecular modeling applications with graphics processors. J Comput Chem 28(16):2618-2640

29. Tang YH, Karniadakis GE (2014) Accelerating dissipative particle dynamics simulations on gpus: algorithms, numerics and applications. Comput Phys Commun 185(11):2809-2822. https://doi. org/10.1016/j.cpc.2014.06.015

30. Trott CR (2011) Lammpscuda-a new gpu accelerated molecular dynamics simulations package and its application to ion-conducting glasses. Ph.d. thesis, Universitätsbibliothek Ilmenau

36. Howard MP, Anderson JA, Nikoubashman A et al (2016) Efficient neighbor list calculation for molecular simulation of colloidal systems using graphics processing units. Comput Phys Commun 203:45-52. https://doi.org/10.1016/j.cpc.2016.02.003

Publisher's Note Springer Nature remains neutral with regard to jurisdictional claims in published maps and institutional affiliations. 